\title{
A Reliable Computational Algorithm for Solving Fractional Biological Population Model
}

\author{
A. Devi ${ }^{*}$, M. Jakhar \\ Department of Mathematics, NIILM University, Kaithal, Haryana-136027, India \\ Received 12 June 2020, accepted in final revised form 11 October 2020
}

\begin{abstract}
In this work, authors obtained the series solution of nonlinear fractional partial differential equations, which is emerging in a spatial diffusion of biological population model using Elzaki transform homotopy perturbation method (ETHPM). The Elzaki transform homotopy perturbation method is a combined form of the Elzaki transform and homotopy perturbation method. Three test illustrations are used to show the proficiency and accuracy of the projected method. It has been observed that the proposed technique can be widely employed to examine other real world problems. The results obtained with the help of the proposed technique are plotted for different fractional orders.
\end{abstract}

Keywords: Biological population model; Elzaki transform; Homotopy perturbation method; Caputo fractional derivative; Mittag-Leffler function.

(C) 2021 JSR Publications. ISSN: 2070-0237 (Print); 2070-0245 (Online). All rights reserved. doi: http://dx.doi.org/10.3329/jsr.v13i1.47521 J. Sci. Res. 13 (1), 59-71 (2021)

\section{Introduction}

Fractional calculus is an integral branch of mathematics for arbitrary derivatives and integrals. Fractional differential equations are vitally important due to their proved and diverse uses in engineering and every part of science. Due to frequent appearance of fractional differential equations in different disciplines of engineering and science, the researchers have added a lot of research contribution to both theory of mathematical science and technology [1-5]. Fractional differential equations are the best for modeling of various processes in engineering and physical sciences. Models allow a better understanding of how complex interactions and processes work. Modeling of dynamic interactions in nature can provide a manageable way of understanding how numbers change over time or in relation to each other. Many models based on real-life phenomena are available [6-8]. Nonlinear phenomena have important effects on applied mathematics, biology, physics and engineering; many physical phenomena are modelled in terms of

* Corresponding author: anju.gill11@gmail.com 
nonlinear partial differential equations. For example, the spatial diffusion of some biological species is described by nonlinear partial differential equations, as

$\frac{\partial}{\partial t} u(x, y, t)=\frac{\partial^{2}}{\partial x^{2}} u^{2}(x, y, t)+\frac{\partial^{2}}{\partial y^{2}} u^{2}(x, y, t)+f(u), \quad t \geq 0, x, y \in R$

With initial condition $u(x, y, 0)=u_{0}(x, y)$, where $u$ denotes the population density and $f$ represents the supply of population (births and deaths in that region). Here, the model is considered as an example in the population of animals. The movements are made generally either by mature animals driven out by invaders or by young animals just reaching maturity moving out of their parental territory to establish breeding territory of their own. In both cases, it is much more plausible to suppose that they will be directed towards nearby vacant territory. Therefore, movement will take place almost exclusively 'down' the population density gradient and will be much more rapid at high population densities than at low ones. In an attempt to model this situation, they considered a walk through a rectangular grid, in which at each step an animal may either stay at its present location or may move in the direction of lowest population density. Here, we consider the more general form of time fractional biological population model by taking $f(u)=$ $h u^{a}\left(1-r u^{b}\right)$ as

$D_{t}^{\alpha} u(x, y, t)=\frac{\partial^{2}}{\partial x^{2}} u^{2}(x, y, t)+\frac{\partial^{2}}{\partial y^{2}} u^{2}(x, y, t)+h u^{a}\left(1-r u^{b}\right), 0<\alpha \leq 1, t \geq 0, x, y \in R$

Here $D_{t}^{\alpha}=\frac{\partial^{\alpha}}{\partial t^{\alpha}}$ is the Caputo fractional derivative with initial condition $u(x, y, 0)=$ $u_{0}(x, y)$, where $u$ denotes the population density and $f$ represents the population supply due to births and deaths and $h, r, a, b$ are real numbers. This proposed population model is discussed elsewhere in literature [9-12]. In the present paper the series solution of biological population model's equation (2) is obtained using Elzaki transform homotopy perturbation method (ETHPM) [13]. Elzaki transform homotopy perturbation method (ETHPM) is one of the effective and straightforward techniques to solve nonlinear FPDEs.

\section{Preliminary Definitions Required in the Sequel}

In this section, some basic definitions that will be incorporated in this study are presented below:

Definition 1. A real function ( $t), t>0$ is said to be in the space $C_{\mu}$ if $\mu \in R$, there exists a real number $p>\mu$ and the function $f_{1}(t) \in C[0, \infty)$ such that $f(t)=t^{p} f_{1}(t)$.

Moreover, if

$f^{(n)} \in C_{\mu}$, then $f(t)$ is said to be in the space $C_{\mu}^{n}, n \in N$.

Definition 2. The Riemann-Liouville fractional integral of order $\alpha \geq 0$ for a function $f(t)$ is defined as

$\mathrm{I}^{\alpha} f(t)= \begin{cases}\frac{1}{\Gamma(\alpha)} \int_{0}^{t}(t-\tau)^{\alpha-1} f(\tau) d \tau, & \alpha>0 \\ f(t), & \alpha=0\end{cases}$ 
Where $\Gamma(\cdot)$ denotes the Gamma function.

Definition 3. The Riemann-Liouville fractional derivative of order $\alpha>0$ for a function $f(t)$ is defined as

$$
\begin{aligned}
\mathrm{D}^{\alpha} f(t) & =\frac{d^{n}}{d t^{n}} I^{n-\alpha} f(t), \\
& =I^{n-\alpha} \frac{d^{n}}{d t^{n}} f(t), \quad n \in N, n-1<\alpha \leq n .
\end{aligned}
$$

Definition 4. The Caputo fractional derivative of order $\alpha>0$ is defined as

$\mathrm{D}^{\alpha} f(t)=\left\{\begin{array}{lr}\frac{d^{n} f(t)}{d t^{n}}, & \alpha=n, \quad n \in N \\ \frac{1}{\Gamma(\mathrm{n}-\alpha)} \int_{0}^{t} \frac{f^{(n)}(\tau)}{(t-\tau)^{\alpha-n+1}} d \tau, & 0 \leq n-1<\alpha<n,\end{array}\right.$

Where $n$ is an integer, $t>0$ and $f(t) \in C_{1}^{n}$. The details of Definition 1 to Definition 4 are discussed in literature [14-16].

Definition 5. In 1903, the Swedish mathematician Gosta Mittag-Leffler introduced the function $E_{\beta}(z)$ [17], defined as

$E_{\beta}(z)=\sum_{k=0}^{\infty} \frac{z^{k}}{\Gamma(\beta k+1)}$,

Where $z$ is a complex variable, $\Gamma(\cdot)$ denotes the Gamma function and $\beta \geq 0$. The MittagLeffler function is the direct generalization of the exponential function to which it reduces for $\beta=1$.

Definition 6. A new integral transform called Elzaki transform of $f(t)$ is defined [18], as $E[f(t)]=E[f(t), v]=T(v)=v \int_{0}^{\infty} f(t) e^{-\frac{t}{v}} d t, k_{1}<v<k_{2}, k_{1}, k_{2}>0,0 \leq t<\infty$.

Where $f(t)$ is taken from the set $\mathrm{A}$, which is defined as

$\mathrm{A}=\left\{f(t) ; \exists M, k_{j}>0, j=1,2,|f(t)|<M e^{\frac{|t|}{k_{j}}}\right.$, if $\left.t \in(-1)^{j} \times[0, \infty)\right\}$.

Here, constant $\mathrm{M}$ must be finite number, $k_{1}$ and $k_{2}$ may be finite or infinite. The detail about Elzaki transform is already discussed in literature [18-21].

Definition 7. Using duality of Laplace [19], Elzaki transform of the Caputo fractional derivative (3), of order $\alpha>0$, can be obtained and defined as

$E\left[D^{\alpha} f(t), v\right]=\frac{T(v)}{v^{\alpha}}-\sum_{k=0}^{n-1} v^{k-\alpha+2} f^{(k)}(0), \quad n-1<\alpha \leq n$,

In (7), $T(v)$ is the Elzaki transform of the function $f(t)$.

\section{Elzaki Transform Homotopy Perturbation Method (ETHPM)}

To illustrate the basic idea of this method; a general fractional nonlinear, nonhomogeneous partial differential equation is considered by us

$D_{t}^{\alpha} u(x, t)=L u(x, t)+N u(x, t)+f(x, t), \alpha>0$,

Subject to the initial condition

$u(x, 0)=g(x)$.

In (8), $D_{t}^{\alpha}=\frac{\partial^{\alpha}}{\partial t^{\alpha}}$ denotes Caputo fractional derivative operator without loss of generality,

L represents a linear differential operator, $N$ stands for nonlinear differential operator and $f(x, t)$ is the source term.

Taking Elzaki transform on both sides of (8), to get 
$E\left[D_{t}^{\alpha} u(x, t)\right]=E[L u(x, t)]+E[N u(x, t)]+E[f(x, t)]$,

Using the differentiation property of Elzaki transform [18-21] and above initial conditions, it yields

$E[u(x, t)]=v^{\alpha} E[L u(x, t)]+v^{\alpha} E[N u(x, t)]+\phi(x, v)$

Applying the inverse Elzaki transform on both sides of (11), arrive at

$u(x, t)=G(x, t)+E^{-1}\left[v^{\alpha} E[L u(x, t)]+v^{\alpha} E[N u(x, t)]\right]$

Where $G(x, t)$ represents the term arising from the known function $f(x, t)$ and the prescribed initial condition.

Now, the homotopy perturbation method is implemented [22-24],

$u(x, t)=\sum_{n=0}^{\infty} p^{n} u_{n}(x, t)$

And the nonlinear term can be decomposed as

$N[u(x, t)]=\sum_{n=0}^{\infty} p^{n} H_{n}(u)$

Where $H_{n}(u)$ are He's polynomials $[25,26]$ and is given by

$H_{n}\left(u_{0}, u_{1}, \ldots, u_{n}\right)=\frac{1}{n !} \frac{\partial^{n}}{\partial p^{n}}\left[N\left(\sum_{i=0}^{\infty} p^{i} u_{i}\right)\right]_{p=0}, \quad n=0,1,2, \ldots$

By substituting (13) and (14), in (15),

$\sum_{n=0}^{\infty} p^{n} u_{n}(x, t)=G(x, t)+p\left(E^{-1}\left[v^{\alpha} E\left(L \sum_{n=0}^{\infty} p^{n} u_{n}(x, t)+\sum_{n=0}^{\infty} p^{n} H_{n}(u)\right)\right]\right)$,

This is the coupling of the Elzaki transform and the Homotopy perturbation method using He's polynomials. Comparing the coefficients of like powers of $p$ in (16) on both sides, it yields the following approximations as

$p^{0}: u_{0}(x, t)=G(x, t)$

$p^{1}: u_{1}(x, t)=E^{-1}\left(v^{\alpha} E\left[L u_{0}(x, t)+H_{0}(u)\right]\right)$

$p^{2}: u_{2}(x, t)=E^{-1}\left(v^{\alpha} E\left[L u_{1}(x, t)+H_{1}(u)\right]\right)$

$p^{3}: u_{3}(x, t)=E^{-1}\left(v^{\alpha} E\left[L u_{2}(x, t)+H_{2}(u)\right]\right)$

$p^{n}: u_{n}(x, t)=E^{-1}\left(v^{\alpha} E\left[L u_{n-1}(x, t)+H_{n-1}(u)\right]\right)$.

Proceeding in the same manner, the rest components of $u_{n}(x, t)$ can be completely found and the series is thus entirely determined. Finally, obtained the analytical solution $u(x, t)$, as

$u(x, t)=\lim _{N \rightarrow \infty} \sum_{n=0}^{N} u_{n}(x, t)$.

The series solution (18) converges very fast in a very few terms.

\section{Numerical Experiments}

In this section, the effectiveness and the usefulness of Elzaki transform homotopy perturbation method (ETHPM) are demonstrated by solving three examples of timefractional biological population model.

Example 1. Here considered the following time-fractional biological population model's equation

$D_{t}^{\alpha} u(x, y, t)=\frac{\partial^{2}}{\partial x^{2}} u^{2}(x, y, t)+\frac{\partial^{2}}{\partial y^{2}} u^{2}(x, y, t)-u(x, y, t)\left(1+\frac{8}{9} u(x, y, t)\right), 0<\alpha \leq 1$,

Subject to the initial condition 
$u(x, y, 0)=\exp \left(\frac{x+y}{3}\right)$.

Employing Elzaki transform on both sides of (19), arrive at

$\frac{E[u(x, y, t)]}{v^{\alpha}}-\sum_{k=0}^{0} v^{k-\alpha+2} u^{(k)}(x, y, 0)=E\left[\frac{\partial^{2}}{\partial x^{2}} u^{2}(x, y, t)+\frac{\partial^{2}}{\partial y^{2}} u^{2}(x, y, t)-u(x, y, t)-\right.$ $\left.\frac{8}{9} u^{2}(x, y, t)\right]$,

On using initial condition and after that a little simplification, get as

$E[u(x, y, t)]=v^{2} \exp \left(\frac{x+y}{3}\right)+v^{\alpha} E\left[\frac{\partial^{2}}{\partial x^{2}} u^{2}+\frac{\partial^{2}}{\partial y^{2}} u^{2}-u-\frac{8}{9} u^{2}\right]$,

Taking inverse Elzaki transform on both sides of (21), get as

$u(x, y, t)=\exp \left(\frac{x+y}{3}\right)+E^{-1}\left[v^{\alpha} E\left[\frac{\partial^{2}}{\partial x^{2}} u^{2}+\frac{\partial^{2}}{\partial y^{2}} u^{2}-\frac{8}{9} u^{2}-u\right]\right.$,

Now, on applying the homotopy perturbation method [22- 24]

$u(x, y, t)=\sum_{n=0}^{\infty} p^{n} u_{n}(x, y, t)$,

And the nonlinear term can be decomposed on putting

$N[u(x, y, t)]=\sum_{n=0}^{\infty} p^{n} H_{n}(u)$,

Substituting (23) and (24) into (22), the following homotopy is constructed

$\sum_{n=0}^{\infty} p^{n} u_{n}(x, y, t)=\exp \left(\frac{x+y}{3}\right)+p\left(E^{-1}\left[v^{\alpha} E\left[\sum_{n=0}^{\infty} p^{n} H_{n}(u)-\sum_{n=0}^{\infty} p^{n} u_{n}(x, y, t)\right]\right]\right)$,

Where $H_{n}(u)$ are He's polynomials [25-26]. The first few components of He's polynomials are given as

$$
\begin{aligned}
& H_{0}(u)=\frac{\partial^{2}}{\partial x^{2}} u_{0}^{2}+\frac{\partial^{2}}{\partial y^{2}} u_{0}^{2}-\frac{8}{9} u_{0}^{2} \\
& H_{1}(u)=2 \frac{\partial^{2}}{\partial x^{2}}\left(\mathrm{u}_{0} \mathrm{u}_{1}\right)+2 \frac{\partial^{2}}{\partial y^{2}}\left(\mathrm{u}_{0} \mathrm{u}_{1}\right)-\frac{16}{9}\left(\mathrm{u}_{0} \mathrm{u}_{1}\right) \\
& H_{2}(u)=2 \frac{\partial^{2}}{\partial x^{2}}\left(\mathrm{u}_{0} \mathrm{u}_{2}\right)+\frac{\partial^{2}}{\partial x^{2}} u_{1}^{2}+2 \frac{\partial^{2}}{\partial y^{2}}\left(\mathrm{u}_{0} \mathrm{u}_{2}\right)+\frac{\partial^{2}}{\partial y^{2}} u_{1}^{2}-\frac{16}{9}\left(\mathrm{u}_{0} \mathrm{u}_{2}\right)-\frac{8}{9} u_{1}^{2}
\end{aligned}
$$

Computing the coefficients of like power of $p$ on both sides in (25), it yields

$p^{0}: u_{0}(x, y, t)=e^{\left(\frac{x+y}{3}\right)}$,

$p^{1}: u_{1}(x, y, t)=E^{-1}\left[v^{\alpha} E\left[H_{0}(u)-u_{0}(x, y, t)\right]\right]$

$p^{1}: u_{1}(x, y, t)=E^{-1}\left[v^{\alpha} E\left[\frac{\partial^{2}}{\partial x^{2}} u_{0}^{2}+\frac{\partial^{2}}{\partial y^{2}} u_{0}^{2}-\frac{8}{9} u_{0}^{2}-u_{0}(x, y, t)\right]\right]$

After little simplification, arrive at

$p^{1}: u_{1}(x, y, t)=-e^{\left(\frac{x+y}{3}\right)} \frac{t^{\alpha}}{\Gamma(\alpha+1)}$,

Similarly

$$
\begin{aligned}
& p^{2}: u_{2}(x, y, t)=E^{-1}\left[v^{\alpha} E\left[H_{1}(u)-u_{1}(x, y, t)\right]\right] \\
& p^{2}: u_{2}(x, y, t)=E^{-1}\left[v^{\alpha} E\left[2 \frac{\partial^{2}}{\partial x^{2}}\left(\mathrm{u}_{0} \mathrm{u}_{1}\right)+2 \frac{\partial^{2}}{\partial y^{2}}\left(\mathrm{u}_{0} \mathrm{u}_{1}\right)-\frac{16}{9}\left(\mathrm{u}_{0} \mathrm{u}_{1}\right)-u_{1}(x, y, t)\right]\right] \\
& p^{2}: u_{2}(x, y, t)=e^{\left(\frac{x+y}{3}\right)} \frac{t^{2 \alpha}}{\Gamma(2 \alpha+1)}, \\
& \text { Similarly } \\
& p^{3}: u_{3}(x, y, t)=E^{-1}\left[v^{\alpha} E\left[H_{2}(u)-u_{2}(x, y, t)\right]\right] \\
& p^{3}: u_{3}(x, y, t)=E^{-1}\left[v^{\alpha} E\left[2 \frac{\partial^{2}}{\partial x^{2}}\left(\mathrm{u}_{0} \mathrm{u}_{2}\right)+\frac{\partial^{2}}{\partial x^{2}} u_{1}^{2}+2 \frac{\partial^{2}}{\partial y^{2}}\left(\mathrm{u}_{0} \mathrm{u}_{2}\right)+\frac{\partial^{2}}{\partial y^{2}} u_{1}^{2}-\frac{16}{9}\left(\mathrm{u}_{0} \mathrm{u}_{2}\right)-\frac{8}{9} u_{1}^{2}-u_{2}(x, y, t)\right]\right]
\end{aligned}
$$


$p^{3}: u_{3}(x, y, t)=-e^{\left(\frac{x+y}{3}\right)} \frac{t^{3 \alpha}}{\Gamma(3 \alpha+1)}$,

Proceeding in similar manner the rest terms of $u_{n}(x, y, t)$ can be found; substituting above obtained terms in (18), solution of Example 1 will be found, in the form of a series

$u(x, y, t)=e^{\left(\frac{x+y}{3}\right)}\left[1-\frac{t^{\alpha}}{\Gamma(\alpha+1)}+\frac{t^{2 \alpha}}{\Gamma(2 \alpha+1)}-\frac{t^{3 \alpha}}{\Gamma(3 \alpha+1)}+\frac{t^{4 \alpha}}{\Gamma(4 \alpha+1)} \ldots\right]$,

By using (4), it yields

$u(x, y, t)=e^{\left(\frac{x+y}{3}\right)} E_{\alpha}\left(-t^{\alpha}\right)$.

Remark 1. Setting $\alpha=1$, in Example 1, it reduces to integer order nonlinear PDE, as

$\frac{\partial}{\partial t} u(x, y, t)=\frac{\partial^{2}}{\partial x^{2}} u^{2}(x, y, t)+\frac{\partial^{2}}{\partial y^{2}} u^{2}(x, y, t)-u(x, y, t)\left(1+\frac{8}{9} u(x, y, t)\right)$,

With initial condition

$u(x, y, 0)=\exp \left(\frac{x+y}{3}\right)$.

On putting $\alpha=1$ in (26), we directly obtained the solution of (28), as

$u(x, y, t)=e^{\left(\frac{x+y}{3}\right)}\left[1-\frac{t}{\Gamma(2)}+\frac{t^{2}}{\Gamma(3)}-\frac{t^{3}}{\Gamma(4)}+\frac{t^{4}}{\Gamma(5)} \ldots\right]$,

Which converge very rapidly to the exact solution of (28), yields as

$u(x, y, t)=e^{\left(\frac{x+y-3 t}{3}\right)}$.
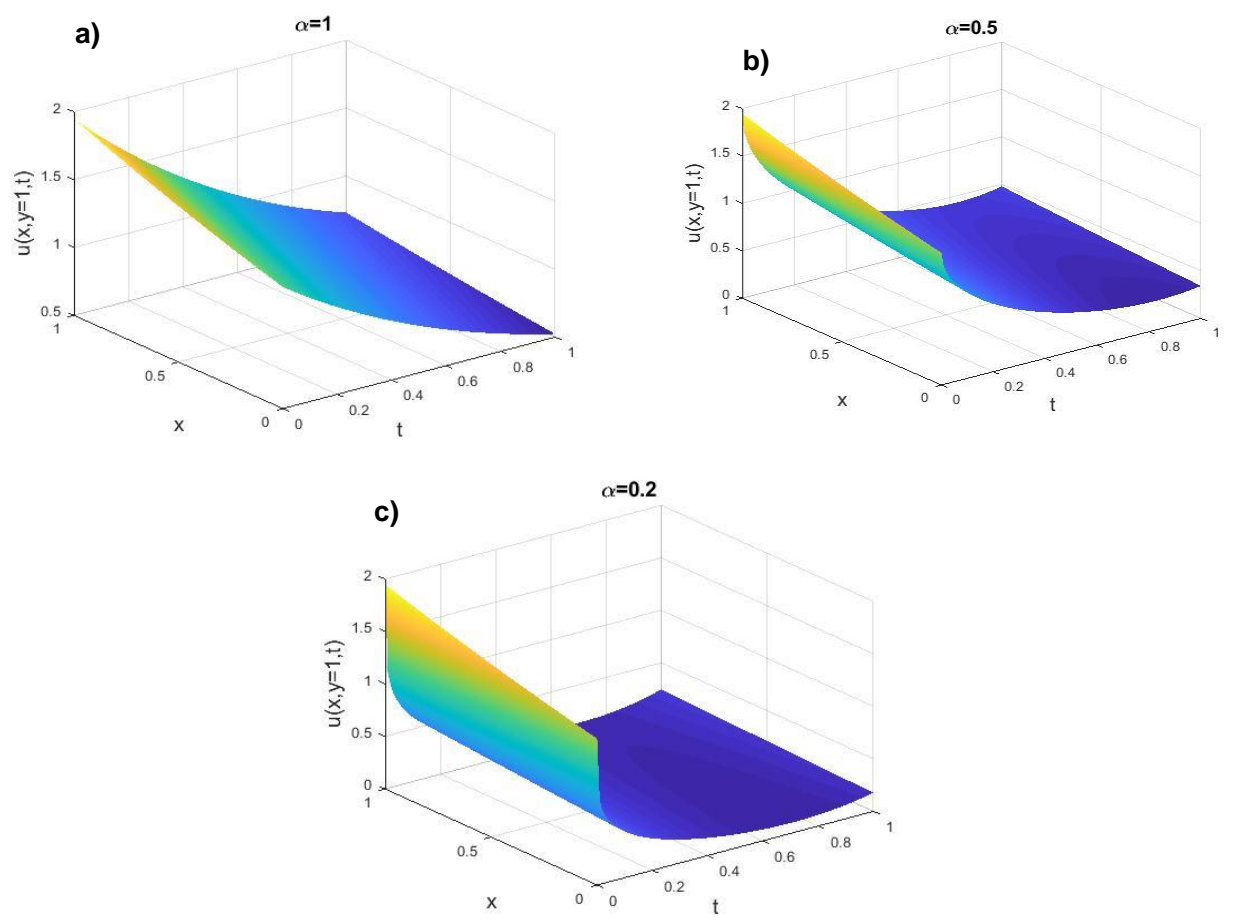

Fig. 1. Surface shows the behavior of solution $u(x, y, t)$ of Example 1 with respect to $x$ and $t$ with $y=1$, when (a) $\alpha=1$; (b) $\alpha=0.5$; (c) $\alpha=0.2$. 


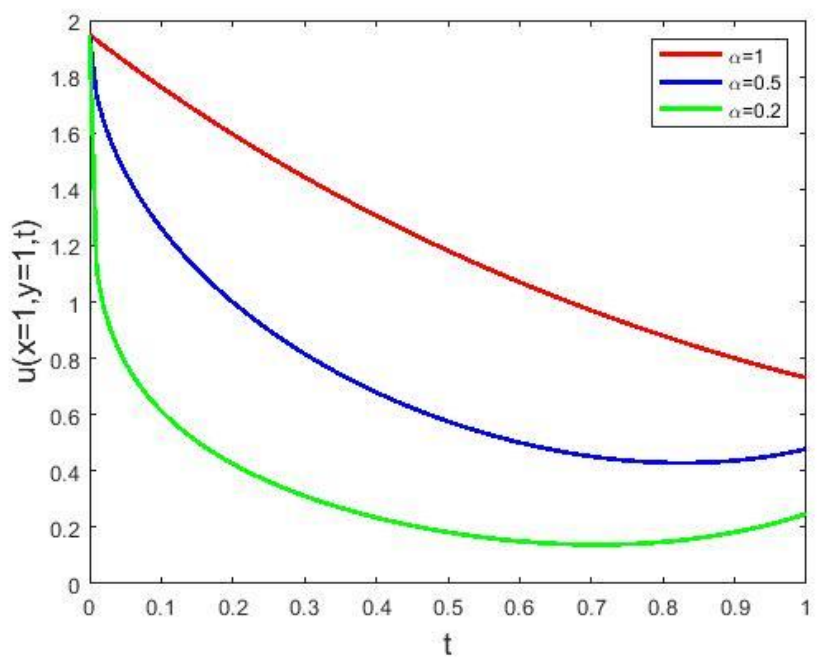

Fig. 2. Plots of $u(x, y, t)$, i. e. solution of Example 1, versus $t$ at $x=1$ and $y=1$ for different fractional orders.

It is observed from Fig. 1a-c that $u(x, y, t)$ increases with the increase in $x$ and decreases with increase in $t$ for $\alpha=1, \alpha=0.5, \alpha=0.2$ with $y=1$. It is also observed from Fig. 2; as the value of $\alpha$ increases, the value of $u(x, y, t)$ also increases.

Example 2. Next, considered the following time-fractional biological population model's equation with initial condition

$D_{t}^{\alpha} u(x, y, t)=\frac{\partial^{2}}{\partial x^{2}} u^{2}(x, y, t)+\frac{\partial^{2}}{\partial y^{2}} u^{2}(x, y, t)+\frac{1}{5} u(x, y, t), \quad 0<\alpha \leq 1$,

$u(x, y, 0)=\sqrt{x y}$.

Incorporating Elzaki transform on (31) with initial condition, after little simplification, arrive at

$E[u(x, y, t)]=v^{2} \sqrt{x y}+v^{\alpha} E\left[\frac{\partial^{2}}{\partial x^{2}} u^{2}(x, y, t)+\frac{\partial^{2}}{\partial y^{2}} u^{2}(x, y, t)+\frac{1}{5} u(x, y, t)\right]$,

Taking inverse Elzaki transform on both sides of (33), get as

$u(x, y, t)=\sqrt{x y}+E^{-1}\left[v^{\alpha} E\left[\frac{\partial^{2}}{\partial x^{2}} u^{2}(x, y, t)+\frac{\partial^{2}}{\partial y^{2}} u^{2}(x, y, t)+\frac{1}{5} u(x, y, t)\right]\right]$,

Further, by applying the Homotopy perturbation technique; as applied in Example 1, get as

$\sum_{n=0}^{\infty} p^{n} u_{n}(x, y, t)=\sqrt{x y}+p\left(E^{-1}\left[v^{\alpha} E\left[\sum_{n=0}^{\infty} p^{n} H_{n}(u)+\frac{1}{5} \sum_{n=0}^{\infty} p^{n} u_{n}(x, y, t)\right]\right]\right)$,

Where $H_{n}(u)$ are He's polynomials (see, [25- 26]). Some components of He's polynomials are represented as

$$
\begin{aligned}
& H_{0}(u)=\frac{\partial^{2}}{\partial x^{2}} u_{0}^{2}+\frac{\partial^{2}}{\partial y^{2}} u_{0}^{2} \\
& H_{1}(u)=2 \frac{\partial^{2}}{\partial x^{2}}\left(\mathrm{u}_{0} \mathrm{u}_{1}\right)+2 \frac{\partial^{2}}{\partial y^{2}}\left(\mathrm{u}_{0} \mathrm{u}_{1}\right)
\end{aligned}
$$


$H_{2}(u)=2 \frac{\partial^{2}}{\partial x^{2}}\left(\mathrm{u}_{0} \mathrm{u}_{2}\right)+\frac{\partial^{2}}{\partial x^{2}} u_{1}^{2}+2 \frac{\partial^{2}}{\partial y^{2}}\left(\mathrm{u}_{0} \mathrm{u}_{2}\right)+\frac{\partial^{2}}{\partial y^{2}} u_{1}^{2}$

Equating the like power of $p,(35)$ yields

$p^{0}: u_{0}(x, y, t)=\sqrt{x y}$,

$p^{1}: u_{1}(x, y, t)=E^{-1}\left[v^{\alpha} E\left[H_{0}(u)+\frac{1}{5} u_{0}(x, y, t)\right]\right]$

$p^{1}: u_{1}(x, y, t)=E^{-1}\left[v^{\alpha} E\left[\frac{\partial^{2}}{\partial x^{2}} u_{0}^{2}+\frac{\partial^{2}}{\partial y^{2}} u_{0}^{2}+\frac{1}{5} u_{0}(x, y, t)\right]\right]$

After little simplification, arrive at

$p^{1}: u_{1}(x, y, t)=\frac{1}{5} \sqrt{x y} \frac{t^{\alpha}}{\Gamma(\alpha+1)}$,

Similarly

$p^{2}: u_{2}(x, y, t)=E^{-1}\left[v^{\alpha} E\left[H_{1}(u)+\frac{1}{5} u_{1}(x, y, t)\right]\right]$

$p^{2}: u_{2}(x, y, t)=E^{-1}\left[v^{\alpha} E\left[2 \frac{\partial^{2}}{\partial x^{2}}\left(\mathrm{u}_{0} \mathrm{u}_{1}\right)+2 \frac{\partial^{2}}{\partial y^{2}}\left(\mathrm{u}_{0} \mathrm{u}_{1}\right)+\frac{1}{5} u_{1}(x, y, t)\right]\right]$

$p^{2}: u_{2}(x, y, t)=\left(\frac{1}{5}\right)^{2} \sqrt{x y} \frac{t^{2 \alpha}}{\Gamma(2 \alpha+1)}$,

Similarly

$p^{3}: u_{3}(x, y, t)=E^{-1}\left[v^{\alpha} E\left[H_{2}(u)+\frac{1}{5} u_{2}(x, y, t)\right]\right]$

$p^{3}: u_{3}(x, y, t)=E^{-1}\left[v^{\alpha} E\left[2 \frac{\partial^{2}}{\partial x^{2}}\left(\mathrm{u}_{0} \mathrm{u}_{2}\right)+\frac{\partial^{2}}{\partial x^{2}} u_{1}^{2}+2 \frac{\partial^{2}}{\partial y^{2}}\left(\mathrm{u}_{0} \mathrm{u}_{2}\right)+\frac{\partial^{2}}{\partial y^{2}} u_{1}^{2}+\frac{1}{5} u_{2}(x, y, t)\right]\right]$

$p^{3}: u_{3}(x, y, t)=\left(\frac{1}{5}\right)^{3} \sqrt{x y} \frac{t^{3 \alpha}}{\Gamma(3 \alpha+1)}$,

Proceeding in similar manner the rest terms of $u_{n}(x, y, t)$ can be found; substituting above obtained terms in (18), solution of Example 2 will be found, in the form of a series

$u(x, y, t)=\sqrt{x y}\left[1+\frac{1}{5} \frac{t^{\alpha}}{\Gamma(\alpha+1)}+\left(\frac{1}{5}\right)^{2} \frac{t^{2 \alpha}}{\Gamma(2 \alpha+1)}+\left(\frac{1}{5}\right)^{3} \frac{t^{3 \alpha}}{\Gamma(3 \alpha+1)}+\left(\frac{1}{5}\right)^{4} \frac{t^{4 \alpha}}{\Gamma(4 \alpha+1)}+\cdots\right]$,

By using (4), it yields

$u(x, y, t)=\sqrt{x y} E_{\alpha}\left(\frac{1}{5} t^{\alpha}\right)$.

Remark 2. Setting $\alpha=1$, in Example 2, it reduces to integer order nonlinear PDE, as

$\frac{\partial}{\partial t} u(x, y, t)=\frac{\partial^{2}}{\partial x^{2}} u^{2}(x, y, t)+\frac{\partial^{2}}{\partial y^{2}} u^{2}(x, y, t)+\frac{1}{5} u(x, y, t)$,

With initial condition

$u(x, y, 0)=\sqrt{x y}$.

On putting $\alpha=1$ in (36), directly obtained the solution of (38), as

$u(x, y, t)=\sqrt{x y}\left[1+\frac{1}{5} \frac{t}{\Gamma(2)}+\left(\frac{1}{5}\right)^{2} \frac{t^{2}}{\Gamma(3)}+\left(\frac{1}{5}\right)^{3} \frac{t^{3}}{\Gamma(4)}+\cdots\right]$,

Which is converges very rapidly to the exact solution of (38), as

$u(x, y, t)=\sqrt{x y} e^{\frac{t}{5}}$. 

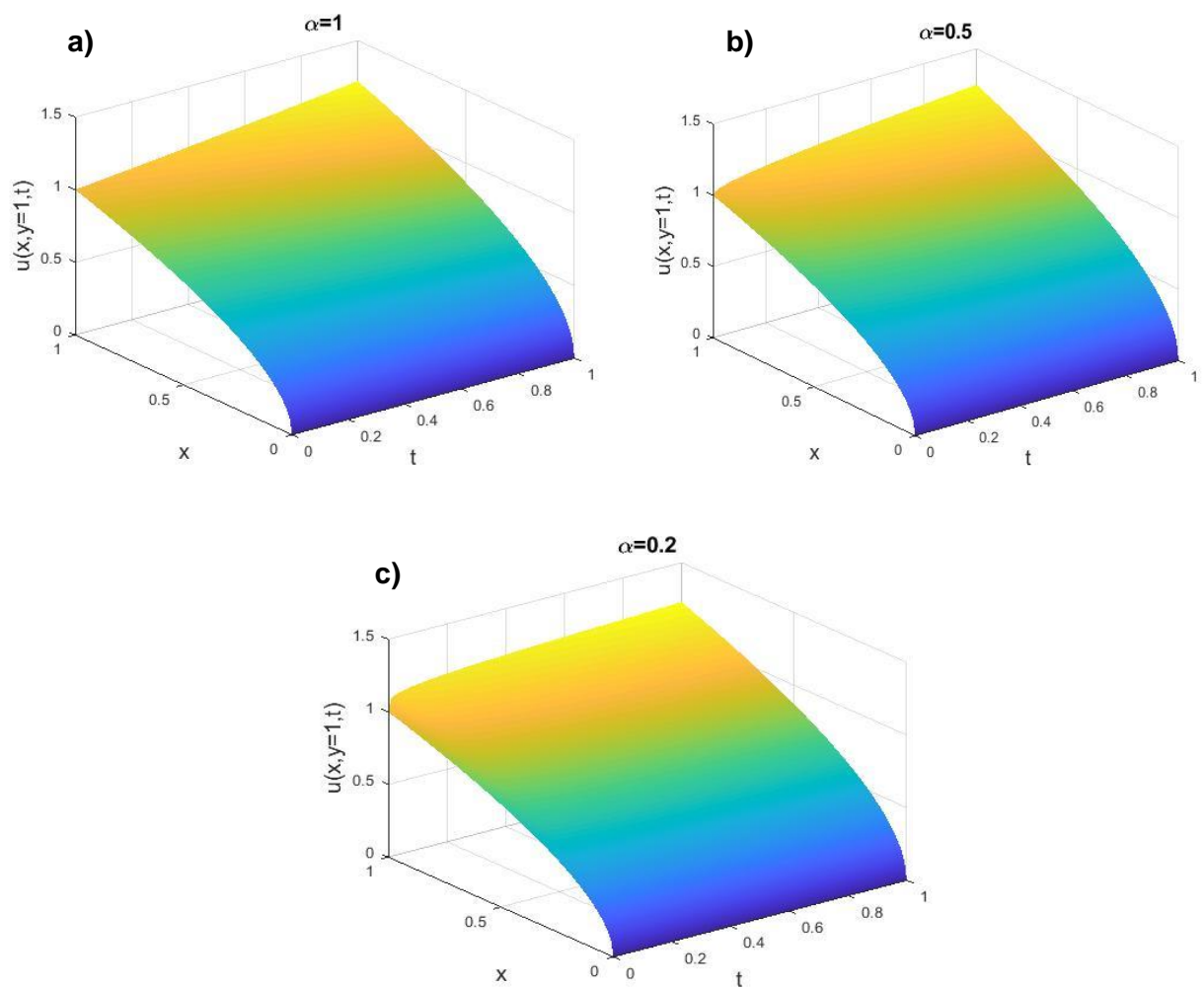

Fig. 3. Surface shows the behavior of solution $u(x, y, t)$ of Example 2 with respect to $x$ and $t$ with $y=1$, when (a) $\alpha=1$; (b) $\alpha=0.5$; (c) $\alpha=0.2$.

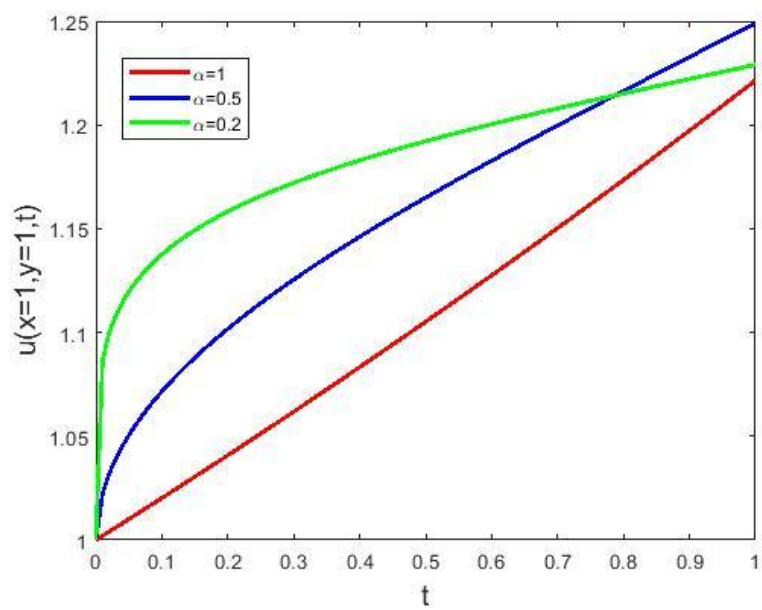

Fig. 4. Plots of $u(x, y, t)$, i. e. solution of Example 2, versus $t$ at $x=1$ and $y=1$ for different fractional orders. 
It is observed from Fig. 3a-c that $u(x, y, t)$ increases with the increase in both $x$ and $t$ for $\alpha=1, \alpha=0.5, \alpha=0.2$ with $y=1$. It is also observed from Fig. 4; as the value of $\alpha$ decreases, the numerical value of $u(x, y, t)$ increases but afterward its nature is slightly change.

Example 3. Finally, considered the following time-fractional biological population model's equation with initial condition

$D_{t}^{\alpha} u(x, y, t)=D_{x}^{2} u^{2}(x, y, t)+D_{y}^{2} u^{2}(x, y, t)+u(x, y, t), \quad 0<\alpha \leq 1$,

$u(x, y, 0)=\sqrt{\sin x \sinh y}$.

Incorporating Elzaki transform on (41) with initial condition, after little simplification, we get

$E[u(x, y, t)]=v^{2} \sqrt{\sin x \sinh y}+v^{\alpha} E\left[\frac{\partial^{2}}{\partial x^{2}} u^{2}(x, y, t)+\frac{\partial^{2}}{\partial y^{2}} u^{2}(x, y, t)+u(x, y, t)\right]$,

Taking inverse Elzaki transform on both sides of (43), get as

$u(x, y, t)=\sqrt{\sin x \sinh y}+E^{-1}\left[v^{\alpha} E\left[\frac{\partial^{2}}{\partial x^{2}} u^{2}(x, y, t)+\frac{\partial^{2}}{\partial y^{2}} u^{2}(x, y, t)+u(x, y, t)\right]\right]$,

Further, employing Homotopy perturbation technique [14-16] as applied in Example 1 and construct the following homotopy

$\sum_{n=0}^{\infty} p^{n} u_{n}(x, y, t)=$

$\sqrt{\sin x \sinh y}+p\left(E^{-1}\left[v^{\alpha} E\left[\sum_{n=0}^{\infty} p^{n} H_{n}(u)+\sum_{n=0}^{\infty} p^{n} u_{n}(x, y, t)\right]\right]\right)$,

Where $H_{n}(u)$ are He's polynomials (see, [25,26]). Some components of He's polynomials are represented as

$$
\begin{aligned}
& H_{0}(u)=\frac{\partial^{2}}{\partial x^{2}} u_{0}^{2}+\frac{\partial^{2}}{\partial y^{2}} u_{0}^{2} \\
& H_{1}(u)=2 \frac{\partial^{2}}{\partial x^{2}}\left(\mathrm{u}_{0} \mathrm{u}_{1}\right)+2 \frac{\partial^{2}}{\partial y^{2}}\left(\mathrm{u}_{0} \mathrm{u}_{1}\right) \\
& H_{2}(u)=2 \frac{\partial^{2}}{\partial x^{2}}\left(\mathrm{u}_{0} \mathrm{u}_{2}\right)+\frac{\partial^{2}}{\partial x^{2}} u_{1}^{2}+2 \frac{\partial^{2}}{\partial y^{2}}\left(\mathrm{u}_{0} \mathrm{u}_{2}\right)+\frac{\partial^{2}}{\partial y^{2}} u_{1}^{2}
\end{aligned}
$$

Comparing the like power of $p,(45)$ yields

$p^{0}: u_{0}(x, y, t)=\sqrt{\sin x \sinh y}$,

$p^{1}: u_{1}(x, y, t)=E^{-1}\left[v^{\alpha} E\left[H_{0}(u)+u_{0}(x, y, t)\right]\right]$

$p^{1}: u_{1}(x, y, t)=E^{-1}\left[v^{\alpha} E\left[\frac{\partial^{2}}{\partial x^{2}} u_{0}^{2}+\frac{\partial^{2}}{\partial y^{2}} u_{0}^{2}+u_{0}(x, y, t)\right]\right]$

After little simplification, arrive at

$p^{1}: u_{1}(x, y, t)=\sqrt{\sin x \sinh y} \frac{t^{\alpha}}{\Gamma(\alpha+1)}$,

Similarly

$p^{2}: u_{2}(x, y, t)=E^{-1}\left[v^{\alpha} E\left[H_{1}(u)+u_{1}(x, y, t)\right]\right]$

$p^{2}: u_{2}(x, y, t)=E^{-1}\left[v^{\alpha} E\left[2 \frac{\partial^{2}}{\partial x^{2}}\left(\mathrm{u}_{0} \mathrm{u}_{1}\right)+2 \frac{\partial^{2}}{\partial y^{2}}\left(\mathrm{u}_{0} \mathrm{u}_{1}\right)+u_{1}(x, y, t)\right]\right]$

$p^{2}: u_{2}(x, y, t)=\sqrt{\sin x \sinh y} \frac{t^{2 \alpha}}{\Gamma(2 \alpha+1)}$,

Similarly

$p^{3}: u_{3}(x, y, t)=E^{-1}\left[v^{\alpha} E\left[H_{2}(u)+u_{2}(x, y, t)\right]\right]$ 
$p^{3}: u_{3}(x, y, t)=E^{-1}\left[v^{\alpha} E\left[2 \frac{\partial^{2}}{\partial x^{2}}\left(\mathrm{u}_{0} \mathrm{u}_{2}\right)+\frac{\partial^{2}}{\partial x^{2}} u_{1}^{2}+2 \frac{\partial^{2}}{\partial y^{2}}\left(\mathrm{u}_{0} \mathrm{u}_{2}\right)+\frac{\partial^{2}}{\partial y^{2}} u_{1}^{2}+u_{2}(x, y, t)\right]\right]$ $p^{3}: u_{3}(x, y, t)=\sqrt{\sin x \sinh y} \frac{t^{3 \alpha}}{\Gamma(3 \alpha+1)}$,

Proceeding in similar manner the rest terms of $u_{n}(x, y, t)$ can be found; substituting above obtained terms in (18), solution of Example 3 will be found, in the form of a series $u(x, y, t)=\sqrt{\sin x \sinh y}\left[1+\frac{t^{\alpha}}{\Gamma(\alpha+1)}+\frac{t^{2 \alpha}}{\Gamma(2 \alpha+1)}+\frac{t^{3 \alpha}}{\Gamma(3 \alpha+1)}+\frac{t^{4 \alpha}}{\Gamma(4 \alpha+1)}+\cdots\right]$,

By using (4), it yields

$u(x, y, t)=\sqrt{x y} E_{\alpha}\left(t^{\alpha}\right)$.

Remark 3. Setting $\alpha=1$, in Example 3, it reduces to integer order nonlinear PDE, as

$\frac{\partial}{\partial t} u(x, y, t)=\frac{\partial^{2}}{\partial x^{2}} u^{2}(x, y, t)+\frac{\partial^{2}}{\partial y^{2}} u^{2}(x, y, t)+u(x, y, t)$,

With initial condition

$u(x, y, 0)=\sqrt{\sin x \sinh y}$.

On putting $\alpha=1$ in (46), we directly obtained the solution of (48), as

$u(x, y, t)=\sqrt{\sin x \sinh y}\left[1+\frac{t}{\Gamma(2)}+\frac{t^{2}}{\Gamma(3)}+\frac{t^{3}}{\Gamma(4)}+\cdots\right]$,

Which converge very rapidly to the exact solution of (48), yields as

$u(x, y, t)=\sqrt{\sin x \sinh y} e^{t}$.
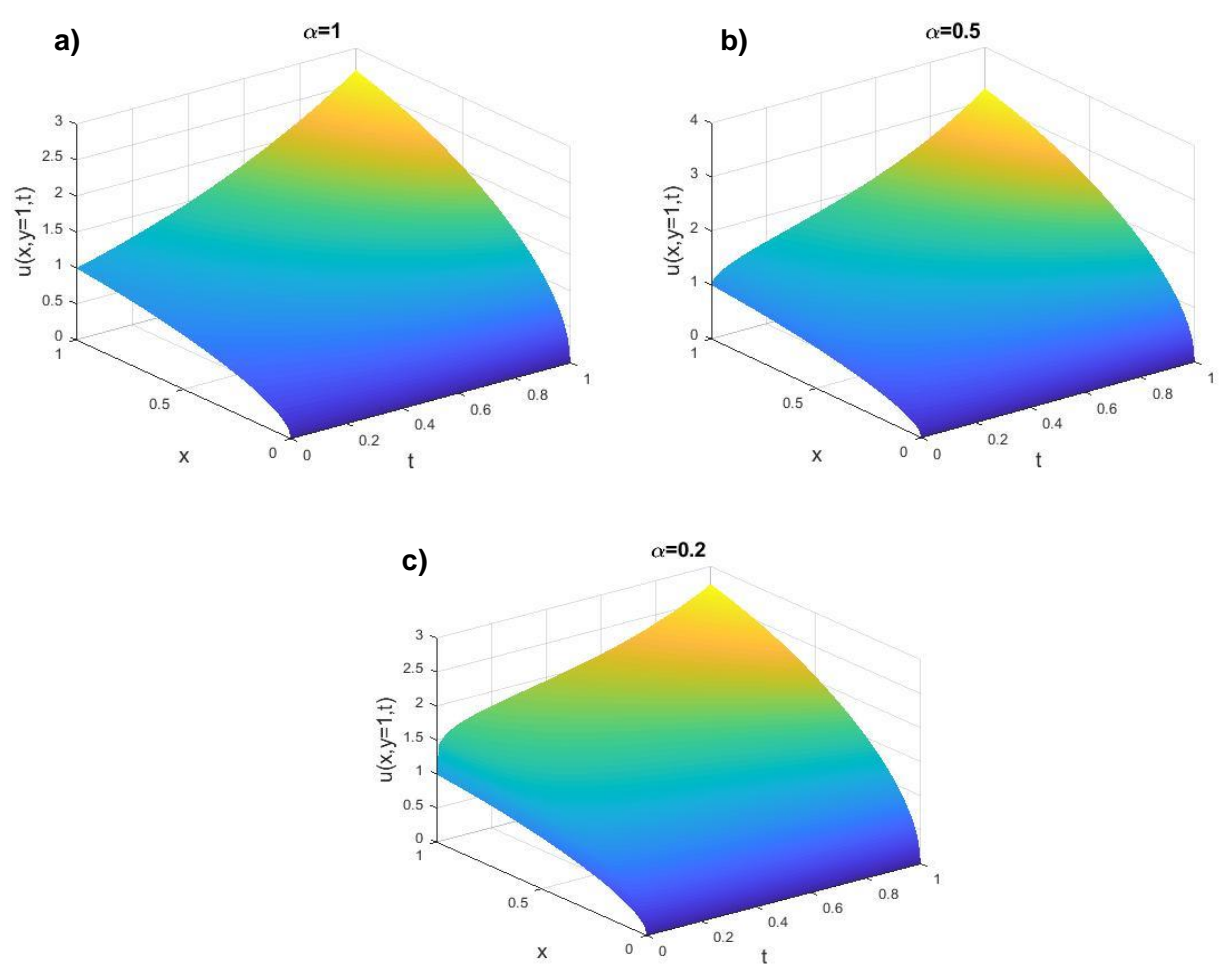

Fig. 5. Surface shows the behavior of solution $u(x, y, t)$ of Example 3 with respect to $x$ and $t$ with $y=1$, when (a) $\alpha=1$; (b) $\alpha=0.5$; (c) $\alpha=0.2$ 


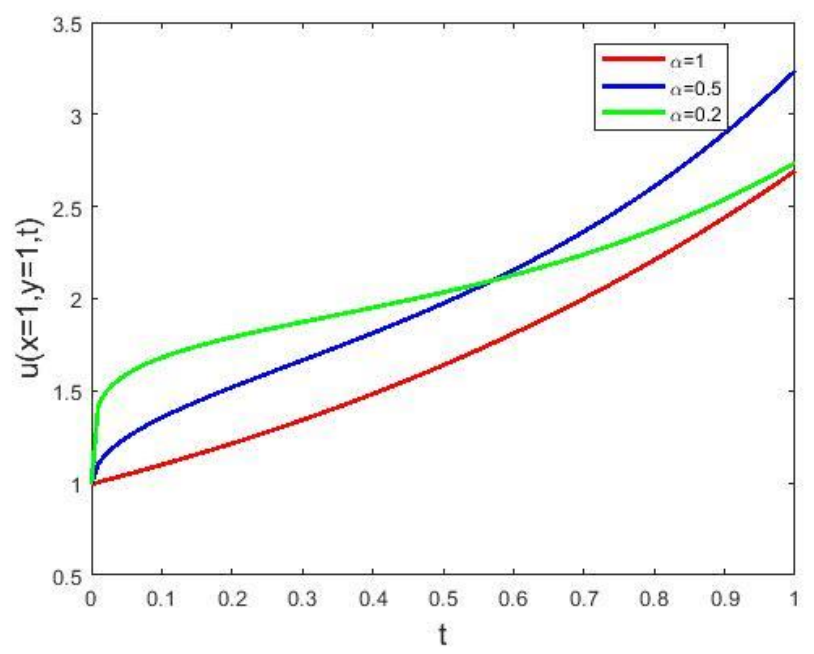

Fig. 6. Plots of $u(x, y, t)$, i. e. solution of Example 3, versus $t$ at $x=1$ and $y=1$ for different fractional orders.

It is observed from Fig. 5a-c that $u(x, y, t)$ increases with the increase in both $x$ and $t$ for $\alpha=1, \alpha=0.5, \alpha=0.2$ with $y=1$. It is also observed from Fig. 6; as the value of $\alpha$ decreases, the numerical value of $u(x, y, t)$ increases but afterward its nature is slightly change.

\section{Conclusion}

The main goal of this paper is to show the applicability of the mixture of new integral transform "Elzaki transform" with the homotopy perturbation method to obtain the solutions in terms of convergent series for nonlinear time-fractional biological population model's equation. The complete deportment of population density is shown in different plotted graphs. The numerical result shows that the proposed method (ETHPM) is very simple and straight forward to implement. The numerical results obtained with the proposed techniques are in an excellent agreement with the exact solution. Hence, it is concluded that the ETHPM is very powerful and efficient in finding analytical as well as exact numerical solutions for wide classes of nonlinear fractional partial differential equations arising in science and engineering.

\section{References}

1. V. Gill and R. S. Dubey, Eur. J. Adv. Eng. Technol. 5, 649 (2018).

2. M. Z. Mohamed and T. M. Elzaki, J. King Saud. Uni. Sci. 32, 544 (2020). https://doi.org/10.1016/j.jksus.2018.08.003

3. Y. Singh, V. Gill, J. Singh, D. Kumar, and K. S. Nisar, Fractal Fract. 4, 33 (2020). https://doi.org/10.3390/fractalfract4030033 
4. V. Gill, K. Modi, and Y. Singh, J. Stat. Manag. Syst. 21, 575 (2018).

https://doi.org/10.1080/09720510.2018.1466966

5. N. B. Manjare and H. T. Dinde, J. Sci. Res. 12, 585 (2020).

https://doi.org/10.3329/jsr.v12i4.47163

6. B. S. Alkahtani, O. J. Algahtani, R. S. Dubey, and P. Goswami, Entropy 19, 114 (2017). https://doi.org/10.3390/e19050114

7. R. S. Dubey and P. Goswami, Discrete Cont. Dynam. Syst. - S (2019). https://doi.org/10.3934/dcdss.2020144

8. I. Haq and I. Singh, J. Sci. Res. 12, 289 (2020). https://doi.org/10.3329/jsr.v12i3.44287

9. M. E. Gurtin and R. C. MacCamy, Math. Biosci. 33, 35 (1977). https://doi.org/10.1016/00255564(77)90062-1

10. W. S. C. Gurney and R.M. Nisbet, J. Theor. Biol. 5, 441 (1975). https://doi.org/10.1016/00225193(75)90011-9

11. Y. G. Lu, Appl. Math. Lett. 13, 123 (2000). https://doi.org/10.1016/S0893-9659(00)00066-5

12. F. Shakeri and M. Dehghan, Comp. Math. Appl. 54, 1197 (2007). https://doi.org/10.1016/j.camwa.2006.12.076

13. P. R. Bhadane and K. P. Ghadle, J. Basic Appl. Res. Int. 20, 29 (2017).

14. A. A. Kilbas, H. M. Srivastava, and J. J. Trujillo, Theory and Applications of Fractional Differential Equations (Elsevier, Netherlands, 2006).

15. I. Podlubny, Fractional Differential Equations (Academic Press, California, USA, 1999).

16. K. S. Miller and B. Ross, An Introduction to the Fractional Calculus and Fractional Differential Equations (John Wiley and Sons, New York, 1993).

17. R. Gorenflo, A. A. Kilbas, and S. V. Rogosin, Integ. Transforms Spec. Funct. 7, 215 (1998). https://doi.org/10.1080/10652469808819200

18. T. M. Elzaki, Glob. J. Pure Appl. Math. 7, 57 (2011).

19. T. M. Elzaki and S. M. Elzaki, Adv. Theor. Appl. Maths. 6, 1 (2011).

20. T. M. Elzaki and S. M. Elzaki, Adv. Theor. Appl. Maths. 6, 13 (2011).

21. H. Kim, Int. J. Math. Anal. 8, 481 (2014).

22. J. H. He, Comput. Methods Appl. Mech. Eng. 178, 257 (1999). https://doi.org/10.1016/S00457825(99)00018-3

23. N. H. Sweilam and M. M. Khader, Comput. Math. Appl. 58, 2134 (2009). https://doi.org/10.1016/j.camwa.2009.03.059

24. J. Saberi-Nadjafi and A. Ghorbani, Comput. Math. Appl. 58, 2379 (2009). https://doi.org/10.1016/j.camwa.2009.03.032

25. A. Ghorbani, Beyond Adomian's Polynomials: He Polynomials, Chaos Solitons Fractals 39, 1486 (2009). https://doi.org/10.1016/j.chaos.2007.06.034

26. S. T. Mohyud-Din, M. A. Noor, and K. I. Noor, Int. J. Nonlinear Sci. Numer. Simul. 10, 227 (2009). https://doi.org/10.1515/IJNSNS.2009.10.2.227 